\title{
Effect of electro-acupuncture on ovarian function of women with diminished ovarian reserve: study protocol for a randomized controlled trial
}

\author{
Le Yang ${ }^{1}$, Hanwang Zhang ${ }^{1}$, Li Zhou², Ying Gao ${ }^{3}$, Lijuan Yang ${ }^{4}$, Yajun Hu², Lu Xu ${ }^{4}$ and Dongmei Huang ${ }^{1 *}$
}

\begin{abstract}
Background: Diminished ovarian reserve (DOR) is the precursor state of ovarian failure and can cause the decline of women's reproductive function. DOR also leads to poor outcome of in vitro fertilization and embryo transfer (IVFET) by affecting the oocytes, high qualified embryo rate, pregnancy rate, etc. Some studies have demonstrated that acupuncture can improve ovarian function. But to date, there is limited evidence indicating that acupuncture or electro-acupuncture is efficient to DOR. This trial aims to evaluate the efficiency and safety of electro-acupuncture for the ovarian function and the following outcome of IVF-ET in DOR patients.
\end{abstract}

Methods: This will be a multicenter randomized controlled clinical trial. A total of more than 338 women with DOR will be randomly allocated to treatment and control groups in 1:1 ratio receiving acupuncture before undergoing IVF-ET. The primary outcome will be the clinical pregnancy rate per cycle of IVF-ET after acupuncture. The secondary outcomes will be ovarian reserve function, outcomes of IVT-ET, blood biochemical index, Massachusetts General Hospital Acupuncture Sensation Scale (MASS), scores from the self-rating anxiety and depression scale, quality of life, and pregnancy outcomes. The safety of acupuncture will also be assessed.

Discussion: The results of this trial may provide high-quality evidence regarding the effectiveness of electroacupuncture in the treatment of DOR and following outcomes of IVF-ET. This will also help patients with DOR and their physicians by offering a new treatment option.

Trial registration: Chinese Clinical Trial Registry ChiCTR1900024626. Registered on 19 July 2019.

Keywords: Electro-acupuncture, Diminished ovarian reserve, In vitro fertilization-embryo transfer, Randomized controlled trial, Study protocol

\section{Background}

Diminished ovarian reserve (DOR) indicates a reduced number of retrieved follicles and the reduction of the quality of oocytes affected by non-biological reasons before the age of 40 . Multiple studies consider that the

\footnotetext{
* Correspondence: hdmjcr@qq.com

${ }^{1}$ Tongji Hospital, Tongji Medical College, Huazhong University of Science and Technology, 1095 Jiefang Avenue, Wuhan 430030, Hubei, China Full list of author information is available at the end of the article
}

cause of DOR is related to age, genetic factors, immune factors, social and environmental factors, infection, iatrogenic injuries, multiple pregnancies, etc. Normally, the ovarian reserve function declines at the age of 30, and fall off sharp after 35 . The incidence rate of DOR is climbing and shows a younger trend. However, there is no effective pharmacotherapy at present.

In-vitro fertilization-embryo transfer (IVF-ET) is a main therapy treating infertility, and controlled ovarian

(C) The Author(s). 2021 Open Access This article is licensed under a Creative Commons Attribution 4.0 International License, which permits use, sharing, adaptation, distribution and reproduction in any medium or format, as long as you give appropriate credit to the original author(s) and the source, provide a link to the Creative Commons licence, and indicate if changes were made. The images or other third party material in this article are included in the article's Creative Commons licence, unless indicated otherwise in a credit line to the material. If material is not included in the article's Creative Commons licence and your intended use is not permitted by statutory regulation or exceeds the permitted use, you will need to obtain permission directly from the copyright holder. To view a copy of this licence, visit http://creativecommons.org/licenses/by/4.0/ The Creative Commons Public Domain Dedication waiver (http://creativecommons.org/publicdomain/zero/1.0/) applies to the data made available in this article, unless otherwise stated in a credit line to the data. 
hyperstimulation $(\mathrm{COH})$ is the key step. In IVF-ET, the success of $\mathrm{COH}$ depends on the ovarian reserve function and ovarian response. A clinical trial finds that when undergoing IVF-ET, the DOR patients' initial dose of gonadotropin (Gn), total Gn, Gn days, and abortion rate are obviously higher than patients with normal ovarian function, but the oocytes, M II oocytes rate, high qualified embryo rate, fertilization rate, implantation rate, and pregnancy rate are all distinct inferior to patients with normal ovarian function [1]. In addition, DOR can cause reproductive endocrine dysfunction including less menstrual volume, menstrual loss, and amenorrhea, which further affects women's reproductive function. Besides, it also leads to perimenopause-related symptoms, such as hot flashes, night sweats, irritability, etc. All these symptoms impact on patients' quality of life. And it will develop into premature ovarian failure (POF) within 1-6 years when without intervention at an early stage. Hence, a new therapy for DOR is necessarily required.

In recent years, as an important part of traditional Chinese medicine, acupuncture has attracted tons of attention in the fields of reproductive endocrine and infertility worldwide [2-4]. Acupuncture can stimulate the neuro-endocrine system, regulate the overall endocrine level and ovarian microenvironment as a whole, improve the internal environment of follicles, improve ovarian hemodynamics, and thereby promote follicular development and ovulation. In addition, acupuncture can also enhance endometrial receptivity and relieve patients' tension and anxiety in assisted reproductive technology (ART) [5]. Acupuncture, which can improve ovarian function, is now widely used in the treatment of POF. Two Meta-analysis show that acupuncture is a relative effective intervention treating POF, which helps for recovering menstruation and boosting level of serum follicle-stimulating hormone (FSH) and estradiol (E2). The curative is sustainable till a month after the end of treatment $[6,7]$. Since DOR can develop to POF within 1-6 months, why do not we intervene it in the early DOR phase in order to delay or reverse the progress to POF?

Given its effect on ovarian function, acupuncture is expected to be a new option for the treatment of DOR. But to date, the available evidence of the efficacy of acupuncture or electro-acupuncture for DOR remains insufficient. Only a few small sample, non-randomized controlled clinical trials suggest that acupuncture or electro-acupuncture can improve ovarian reserve function of DOR patients $[8,9]$. Therefore, a large sample, multicenter, randomized controlled trial is designed to evaluate the efficiency and safety of electro-acupuncture in DOR patients. The clinical pregnancy rate is the primary outcome reflecting ovarian function. The following outcome of IVF-ET will also be evaluated as a secondary outcome.

\section{Objectives}

Through this study, we expect to test the following hypotheses: (1) evaluate whether electro-acupuncture is superior to the control group in ameliorating ovarian function of DOR patients reflected by improving clinical pregnancy rate, and (2) assess the safety of electroacupuncture.

\section{Methods \\ Trial design}

This will be a multicenter, randomized, controlled clinical trial. The trial was designed as a superiority trial, with the aim of evaluating whether the electroacupuncture intervention was superior to the control group in improving ovarian function of DOR patients. A total of more than 338 women with DOR will be randomly allocated to treatment and control groups in 1:1 ratio receiving acupuncture before undergoing IVF-ET.

This trial has been registered at the Chinese Clinical Trial Registry (ChiCTR1900024626). The flowchart and study design schedule are presented in Fig. 1 and Table 1. The Standard Protocol Items: Recommendations for Interventional Trials (SPIRIT) checklist is provided as Additional file 1.

\section{Study setting}

Patients will be recruited from the following 4 hospitals in China: Tongji Hospital affiliated to Tongji Medical College of Huazhong University of Science and Technology, Union Hospital affiliated to Tongji Medical College of Huazhong University of Science and Technology, Wuhan First Hospital, and Reproductive Hospital affiliated to Jiangxi University of Traditional Chinese Medicine.

\section{Consent to participate}

This protocol is in accordance with the principles of the Declaration of Helsinki. Written informed consent will be obtained from patients prior to enrolment. The informed consent is provided as Additional file 2. In each sub-center, two independent gynecologists are responsible for recruiting patients and gaining informed consent. On the consent form, participants will be asked if they agree to the use of their data when they choose to withdraw from the trial. Participants will also be asked for permission for the research team to share relevant data with researchers from the universities taking part in the research or from regulatory authorities, where relevant. This trial does not involve collecting biological 


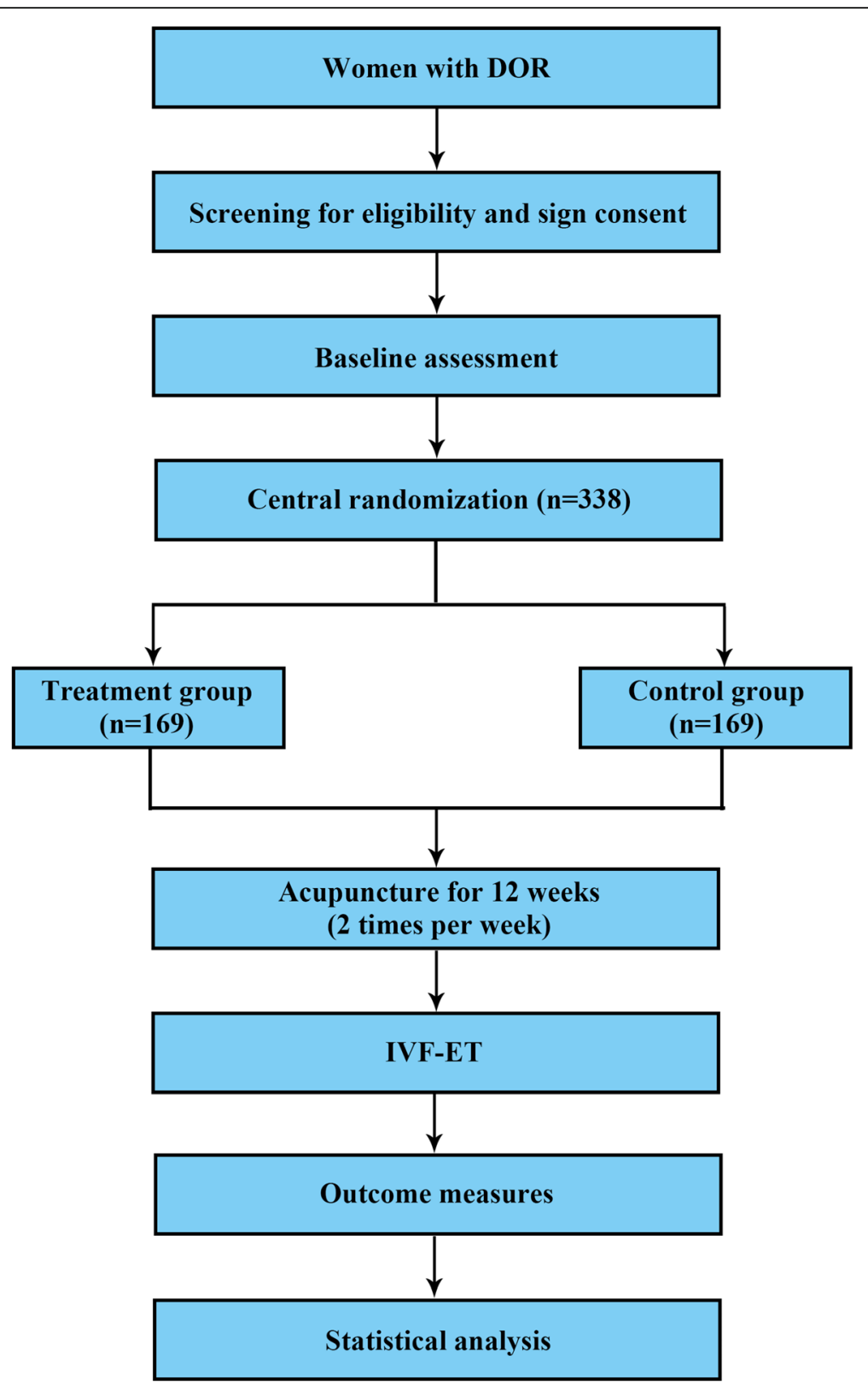

Fig. 1 Flowchart of the trial procedures

specimens for storage. No changes are made to the trial protocol after the subject recruitment started.

\section{Inclusion criteria}

Participants will be included if they meet the following criteria: (1) age < 40, will undergo IVF-ET; (2) low ovarian reserve: antral follicle count (AFC) $<7$, or $\mathrm{AMH}<$ $1.1 \mathrm{ng} / \mathrm{ml}$; or has a history of poor ovarian response: in the last hyperstimulation cycle, the number of retrieved oocytes < 3; (3) spouse' semen examination is normal, or after semen prewash can reach the standard of common IVF or intracytoplasmic sperm injection (ICSI).

\section{Exclusion criteria}

Participants will be excluded if they meet any of the following criteria: (1) male with azoospermia; (2) male/female's chromosome is abnormal; (3) adenomyosis, uterine fibroids, endometrial polyps, scar uterine, reproductive system tuberculosis, oviduct effusion, pelvic lesions such as ovarian endometriosis cyst or tumor; (4) female has other endocrine diseases: thyroid diseases, hyperprolactinemia, insulin resistance, diabetes, adrenal diseases, etc.; (5) definitively diagnosed autoimmune diseases such as systemic lupus erythematosus, rheumatoid arthritis, and antiphospholipid syndrome; (6) other 
Table 1 Participants timeline

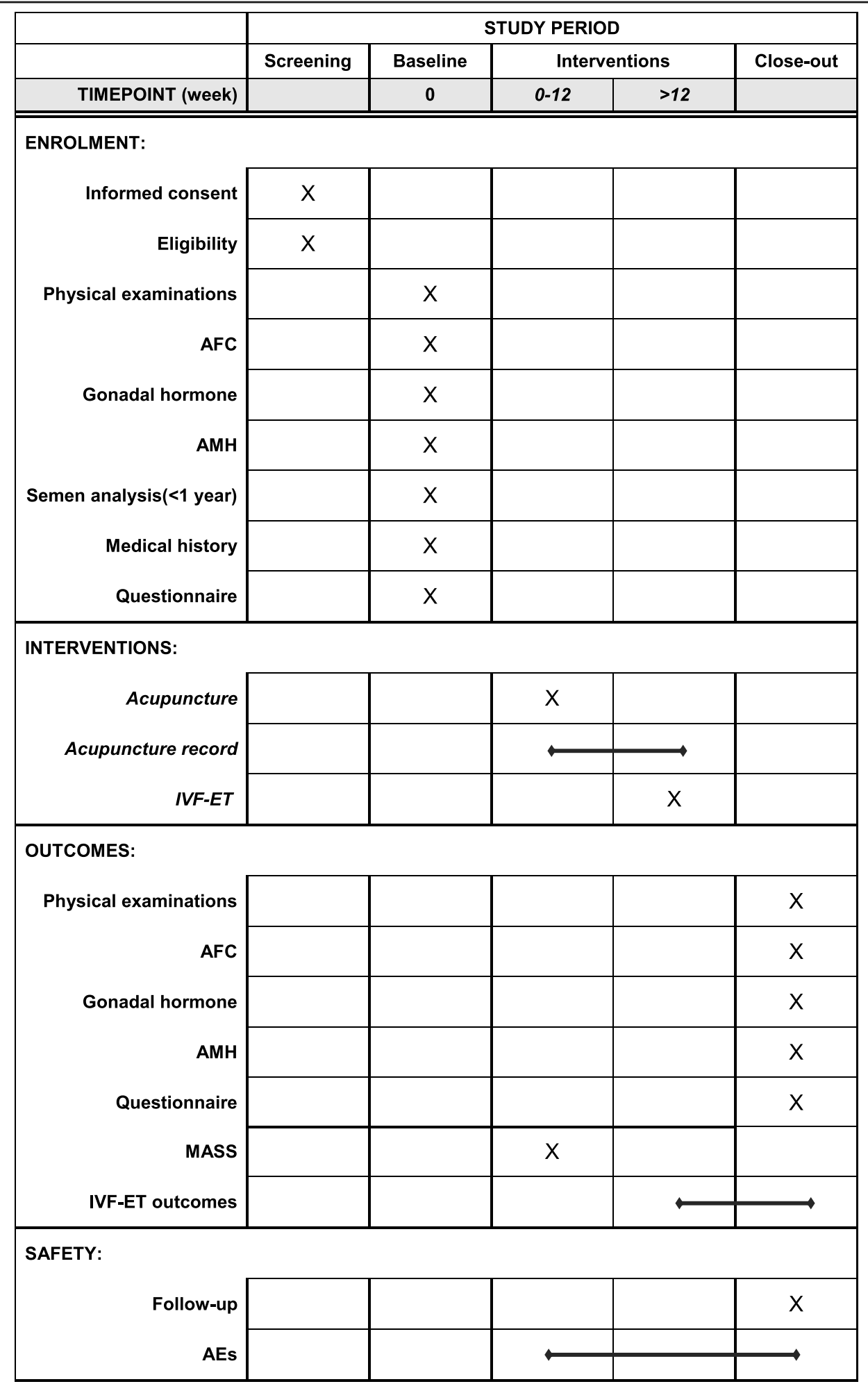

AFC (antral follicle count), AMH (anti-Müllerian hormone), IVF-ET (In-vitro fertilization-embryo transfer), MASS (Massachusetts General Hospital Acupuncture Sensation Scale), AEs (adverse events). 
pathogenesis that leads to recurrent miscarriage or agnogenic recurrent miscarriage; (7) a history of cancer and has received radiotherapy and chemotherapy; (8) had acupuncture treatment in recent 3 months; and (9) unwilling to sign the informed consent of this study.

\section{Randomization and masking}

Internet-based central randomization system will be applied in this trial. Three hundred thirty-eight participants will be randomly allocated to the treatment group and control group in a 1:1 ratio. The Internet-based central randomization system designs the random parameters and allocation. The appointed researchers in each hospital will apply for the group assignment allocated by the system through inputting a specific code. The randomization protocol and the parameters set during it are collectively called blinding code, which are kept strictly by researchers who are not involved in the process of outcome evaluation and statistical analysis.

\section{Interventions}

To improve patients' adherence to interventions, electro-acupuncture will be performed by acupuncturists with over 5 years of clinical acupuncture experience and obtained a license from the Ministry of Health of the People's Republic of China. Before performing the treatment, acupuncturists will receive centralized training including an introduction to the basic clinical research methods and a practical demonstration of the treatment. WeChat will also be used for making appointments and regular reminders. At each part of the study, including assessing ovarian function before and after the intervention, performing electro-acupuncture, or making followup visits, the independent gynecologist will make appointments and remind participants of some notes by using this APP.

The treatments of both two groups start after a spontaneous period or a withdrawal bleeding by progestin. For patients with amenorrhea or oligomenorrhea, the withdrawal bleeding is induced using progesterone by the outpatient doctors, and then the intervention starts after the withdrawal bleeding. The acupuncture protocols are formulated according to the theory of traditional Chinese medicine. The number of needles used, methods of stimulation (manipulation and electroacupuncture), frequency, and time of the treatment are detailed described following the CONSORT and STRICTA recommendations [10].

Fixed protocols are used for both the treatment group and the control group. All patients receive the treatment of active acupuncture or sham acupuncture, twice or three times a week with a maximum of 24 times in 8-12 weeks. Treatments will not be performed during menstruation in both two groups. The treatment date, time, the name of the acupuncturist and the intensity of the electro-acupuncture stimulation which can vary between the different electrodes are recorded when the patient receives acupuncture treatment. The range of the intensity of the electro-acupuncture stimulation will be recorded, e.g., 1.2-3.0 mA.

\section{Treatment group:}

For the treatment group, two groups of acupoints will be used alternatively.

The first group consists of governor vessel (GV) 20, conception vessel $(\mathrm{CV}) 6, \mathrm{CV} 3$, bilateral stomach $(\mathrm{ST})$ 29, bilateral spleen (SP) 6, bilateral ST 36 , and bilateral pericardium (PC) 4 . The patients will be asked to stay in a supine position and keep the whole body relaxed and comfortable. Disposable sterilized needles (size: $0.25 \times$ $40 / 50 \mathrm{~mm}$ ) will be inserted into a depth of $15 \sim 35 \mathrm{~mm}$ and stimulated manually to evoke needle sensation ("Deqi" in traditional Chinese medicine, TCM). And then CV 3 and CV 6, bilateral ST29, and SP6 and SP 9 bilaterally will be thereafter connected to electrical stimulators (Export Abteilung, Schwa-Medico GmbH, Wetzlarer Str. 41-43; 35630 Ehringshausen) and stimulated with low-frequency of $2 \mathrm{~Hz}, 0.3 \mathrm{~ms}$ pulse length for 30 min. The intensity was adjusted to the maximum tolerated intensity of the patients. The other needles without electrical stimulators will be manually stimulated to evoke "Deqi" every $10 \mathrm{~min}$.

The second group of acupoints consists of the bladder (BL) 23 and 32 bilaterally, bilateral kidney (KI) 3 and SP 6 , and GV 20. Patients will be asked to stay in a prone position and keep the whole body relaxed and comfortable. GV 20 will be punctured obliquely, and other acupoints will be perpendicularly needled into a depth of 15 35 mm using disposable sterilized needles (size: 0.25 $\times 40 / 50 \mathrm{~mm}$ ). All acupoints will be stimulated manually to evoke needle sensation ("Deqi" in TCM). And then BL 23 and 32, and KI 3 and SP 6 bilaterally will be stimulated with low-frequency in the same way as the first group. GV 20 will be stimulated manually to evoke "Deqi" every $10 \mathrm{~min}$.

\section{Control group}

For the control group, four pseudo-acupoints are used, with two points on each shoulder and the two on each upper arm, which are not located on any meridians. The patients will be asked to stay in a supine position and keep the whole body relaxed and comfortable. Disposable sterilized needles (Size: $0.18 \times 25 \mathrm{~mm}$ ) will be inserted superficially to a depth of $<5 \mathrm{~mm}$ without any manual stimulus and the needle sensation ("Deqi" in TCM) should not be evoked. Electrodes are connected to the needles, but the stimulators should be turned on 
at an intensity of zero. Each intervention lasts for 30 $\min$.

\section{IVF-ET treatment}

After the acupuncture treatment, patients' ovarian reserve function will be evaluated again, and then IVF-ET cycle treatment will be conducted. This will be performed by the reproductive medicine center according to the patients' situation and the standard procedure.

\section{Concomitant treatments}

Drugs and other treatments, which may interface the evaluation of electro-acupuncture effect, will be discouraged. Discouraged treatments include sexual hormones, contraceptives, and herbs. If treatment not recommended in this trial has already been performed, relevant information should be recorded in the patient's case report form.

\section{Outcome measures}

\section{Primary outcome measure}

The primary outcome is clinical pregnancy rate per cycle of IVF-ET after acupuncture (through study completion, an average of 1 year). Clinical pregnancy was defined by the presence of a fetal heartbeat at 6-7 weeks of pregnancy. The clinical pregnancy rate is the number of clinical pregnancies per embryo transfer cycles.

\section{Secondary outcome measures}

The change of ovarian reserve function Assessing patients' ovarian reserve function before and after acupuncture intervention (at 0 weeks and up to 12 weeks), including:

(1) The serum AMH, inhibin, and FSH and E2 levels on the third day of menstruation;

(2) AFC.

Outcomes of IVF Outcomes of IVF include:

(1) Gn dosage and usage days;

(2) E2 level and endometrial thickness on human chorionic gonadotropin (HCG) day;

(3) Number of oocytes;

(4) MII oocytes;

(5) Normal fertility rate;

(6) The number of available embryos;

(7) Number of high-quality embryos;

(8) Cycle cancelation rate (including cycle cancelation rate caused by various reasons);

(9) Implantation rate: including fresh periodic implantation rate, per cycle implantation rate and cumulative implantation rate;
(10)Fresh cycle clinical pregnancy rate and cycle cumulative clinical pregnancy rate;

(11)Early, mid, and late pregnancy abortion rate;

(12)Risk of ovarian hypertrophy and incidence of obstetric complications;

(13)FSH, LH, E2, and AMH in follicular fluid; oxidative stress-related indicators such as reactive oxygen species (ROS), superoxide dismutase (SOD) level, etc.;

(14)Live rate: including fresh cycle live rate, cycle live rate and cumulative live rate.

These outcomes will be assessed through study completion, at an average of 1 year.

Blood biochemical index examination before and after acupuncture The level of blood corticotrophinreleasing hormone $(\mathrm{CRH})$, norepinephrine, adrenaline, 5-hydroxytryptamine, beta-aminobutyric acid (GABA), dopamine (DA), and neuro-endorphin will be tested before and after treatment (at 0 weeks and up to 12 weeks).

Deqi sensation scale of acupuncture After each acupuncture treatment, patients will be asked to rate the MASS [11] independently by evaluating 12 acupuncture sensation degrees within $5 \mathrm{~min}$. The higher the weighted total score is, the more obvious is the Deqi sensation degree.

Questionnaires Evaluation of anxiety, depression, and quality of life will be performed before and after treatment (at 0 weeks and up to 12 weeks), including:

(1) Baker anxiety self-rating scale (BAI) and baker depression self-rating scale (BDI-II): higher score indicates higher degree of depression or anxiety [12, 13].

(2) Zung anxiety self-rating scale (Zung-SAS), Zung depression self-rating scale (Zung-SDS) [14, 15].

(3) Quality of life measurement (QOL): Quality of life will be assessed by SF-36 and the Chinese quality of life scale (CHQOL).

Follow-up detection Pregnancy patients will be followed up to the end of pregnancy. Adverse pregnancy outcomes and live birth rates will be recorded. Nonpregnant patients will be followed up for 1 year after treatment, testing the ovarian reserve function, followup treatment, and pregnancy status of the patients within 1 year.

\section{Participant retention}

All of the participants, regardless of which group, will receive health education. Health education includes: (1) 
patients will be encouraged to engage in an appropriate exercise training program, (2) patients will be suggested to continue striving for a healthy diet, and (3) patients will be given relaxing training and psychological counseling. For pregnant patients, 3D ultrasound will be appointed in advance and advices during pregnancy and perinatal will be given by gynecologists. Non-pregnant patients will also be followed up for 1 year after treatment and given advices about following treatment like ovulation-inducing scheme of IVF-ET.

\section{Adverse events}

Adverse events (AEs) will be classified. AEs related with electro-acupuncture include bleeding after needle withdrawal, striking of needle, bending of needle, and fainting during acupuncture treatment. AEs related to IVFET include ovarian hyperstimulation syndrome, colporrhagia and infection caused by egg retrieval, and multiple pregnancy. Researchers will obtain AEs by inquiring of the participants at each treatment or followup visit or by voluntarily reporting by the participants. The percentage of AEs and severe adverse events that occurred during treatment will be recorded in detail. Chi-square test is used to analyze the total proportion of adverse events in each treatment regimen and the differences between the classifications. Unless otherwise formally requested, each report from the safety monitor board will report details and summaries of adverse events in a double-blind manner.

\section{Criteria for discontinuing interventions}

Acupuncture will be ended if participants meet any of the following conditions: (1) serious adverse events occurred during or after treatment, (2) misdiagnosed after randomization, and (3) patients found to be pregnant after randomization.

\section{Provisions for post-trial care}

There is no anticipated harm and compensation for trial participation.

\section{Sample size}

According to studies conducted by Hong SB et al. and Roustan A et al., the clinical pregnancy rate of DOR patients is respectively $20.6 \%$ and $20.2 \%$ [16, 17]. Due to the lack of effective randomized blinded controlled clinical trials that choose clinical pregnancy rate per cycle as the primary outcome measure in evaluating acupuncture efficacy, we presume $15 \%$ for the patient's clinical pregnancy rate per cycle. Assuming that the clinical pregnancy rate can be increased by $15 \%$ after acupuncture treatment, $\alpha=0.05, \beta=0.20$, power is $80 \%$. The sample size is calculated according to the following formula [18], $\mathrm{nA}=\mathrm{knB}, \mathrm{nB}=[\mathrm{pA}(1-\mathrm{pA}) / \kappa+\mathrm{pB}(1-\mathrm{pB})](\mathrm{z} 1-\alpha / 2+\mathrm{z} 1-$ $\beta) 2 /(p A-p B) 2, \kappa=1$, then we get $n A=n B=135$, which means the sample size of each group is 135 . Allowing for a $20 \%$ dropout rate, the sample size of this trial is 338 participants.

\section{Recruitment}

Eligible patients will be recruited from participating hospitals through the following strategies. Posters will be placed on doctors' offices, bulletin boards, or other places in the hospitals. Advertisements are also put via network, Wechat, etc. Eligible patients from the outpatient and inpatient clinics will be advised by doctors. In each hospital, specialized staff will contact patients and make an appointment for a screening visit.

\section{Blinding}

In this study, participants, outcome assessors, and statisticians are blinded. The acupuncturists will not be blinded.

Many efforts will be done to maintain blinding in participants assigned to the intervention and control groups. For example, eligible patients will be acupuncture naïve. In the control group, electrodes are connected to the needles, which is the same as the intervention group, but the stimulators should be turned on at an intensity of zero.

We will also try to ensure that the outcome assessors and statisticians are unaware of the group allocation. The two groups will be labeled as groups 1 and 2. Assessors will be randomly assigned to follow-up. Allocation sequence will be concealed until the end of the study. In addition, we will separately give relevant data of AEs, and AES to an independent statistician for analysis, while the statistician responsible for the analysis of primary outcome and other secondary outcomes will be blinded to group allocation.

After the patients completed the whole visit, they will be told which group they were allocated in.

\section{Data management and quality control}

Researchers including acupuncturists, outcome assessors, and statisticians will receive training about data management. In this trial, online monitoring will be used. Data of participants will be inputted into the electronic case report form (eCRF) through clinical trial management public platform called ResMan. The clinical research associates are responsible for verifying the accuracy of data. All the research documents, which include both the paper files and electronic documents, will be preserved for at least five years after publication. The private information of patients (name, telephone number, and ID number) will be anonymous to ensure participant confidentiality. After approval of a proposal and with a signed data access agreement, researchers whose 
proposed use of the data has been approved can have access to the data.

The quality controller of each hospital will complete self-inspection at least once every month. Remote or onsite monitoring will be performed for all centers once per 3 months by the principle investigator. The auditing will be done by DMC (Clinical Research Center of Tongji hospital) at the beginning, middle, and end of the trial. The DMC was established in 2018, which consists of investigators, data managers, biostatisticians, and study coordinators. The DMC is independent of the study organizers. The responsibility of DMS is periodically reviewing the accumulating data and determining if this trial should be modified or discontinued. The DMC does not have executive power; rather, it communicates the outcome of its deliberations to the trial sponsors. The chair is Mr. Zhou JF, with Dr. Xu SB, Dr. Zhu XJ. For patients who deviate from the trial, causes and relevant outcome data will be recorded in a case report form as much as possible.

\section{Statistical methods}

All analyses will be based on the intention-to-treat principle. Data analysis will be performed by an independent statistician using SAS statistical software (version 9.4; SAS Institute Inc.).

The analysis of variance (ANOVA) will be used to test the difference of clinical pregnancy rate (primary outcome) between the two intervention groups with the baseline date as a variate and taking into account the multicenter effect. Missing data will be imputed using the multiple imputation method. Continuous data will be presented as the mean and standard deviation, or the median and interquartile range. Categorical data will be presented as the number and percentage. Comparisons between groups will be analyzed using an independent $t$ test or Wilcoxon ranksum test for continuous variables, and a chi-square test or Fisher exact test for categorical variables. All statistical tests will be two-sided, and $p<0.05$ will be considered statistically significant.

\section{Discussion}

This trial aims to determine whether electroacupuncture can improve ovarian function and clinical pregnancy rate during IVF cycles for DOR patients. Some clinical and experimental studies have found that acupuncture can promote the follicular development and ovulation, and further improve ovarian function [69]. A few clinical trials have shown effectiveness in treating DOR through acupuncture or electro-acupuncture $[8,9,19,20]$. The pregnancy rate in IVF-ET after acupuncture is assessed by several studies [21, 22]. However, the population of these trials are all small sample and non-controlled. Therefore, we will confirm the efficacy by a large sample, multi-center randomized controlled clinical trial. This trial will assess the clinical pregnancy rate as the primary outcome reflecting ovarian function.

In this trial, the form of the electro-acupuncture is manual acupuncture. All acupoints will be stimulated manually to evoke a needle sensation called Deqi. After that, the electrodes will be connected to the needles and start simulating. The intensity of Deqi sensation is critical for the effects of acupuncture [23]. The MASS will be used to quantify the intensity of Deqi sensations after each treatment, which aims to explore the association between the intensity of Deqi sensations and the efficacy of electro-acupuncture.

There are some limitations in this trial. The acupuncture protocol consists of two groups of acupoints, which seems to be a little sophisticated for acupuncturists, and may increase the difficulty in implementing placebo acupuncture. Besides, the acupuncturists in this trial will not be blinded to the intervention. The solution of the situation is blinding the group assignment before electro-acupuncture.

In conclusion, the results of this trial may provide high-quality evidence evaluating the effectiveness of electro-acupuncture in the treatment of DOR patients and following outcomes of IVF-ET. This study will contribute to offer a therapy option for DOR patients.

\section{Trial status}

This trial is currently recruiting participants. The protocol version number and date: V3.0, September 8, 2020. The recruitment began on August 1, 2019. The estimated completion date of recruitment is August 1, 2023.

\section{Abbreviations}

DOR: Diminished ovarian reserve; IVF-ET: In vitro fertilization and embryo transfer; MASS: Massachusetts General Hospital Acupuncture Sensation Scale; $\mathrm{COH}$ : Controlled ovarian hyperstimulation; Gn: Gonadotropin; POF: Premature ovarian failure; ART: Assisted reproductive technology; FSH: Follicle-

stimulating hormone; E2: Estradiol; AFC: Antral follicle count; ICSI: Intracytoplasmic sperm injection; GV: Governor vessel; CV: Conception vessel; ST: Stomach; SP: Spleen; PC: Pericardium; TCM: Traditional Chinese medicine; BL: Bladder; KI: Kidney; HCG: Human chorionic gonadotropin; ROS: Reactive oxygen species; SOD: Superoxide dismutase; $\mathrm{CRH}$ : Corticotrophin-releasing hormone; GABA: Beta-aminobutyric acid; BAI: Baker anxiety self-rating scale; BDI-II: Baker depression self-rating scale; Zung-SAS: Zung anxiety self-rating scale; Zung-SDS: Zung depression selfrating scale; QOL: Quality of life measurement; $\mathrm{CHQOL}$ : Chinese quality of life scale; AEs: Adverse events; eCRF: Electronic case report form

\section{Supplementary Information}

The online version contains supplementary material available at https://doi. org/10.1186/s13063-021-05894-2.

Additional file 1:. SPIRIT checklist

Additional file 2:. Informed consent 


\section{Authors' contributions}

$\mathrm{DMH}$ and $\mathrm{LY}$ conceived and designed the study protocol. LY drafted the manuscript. LY completed the trial registration. DMH and HWZ substantively revised the manuscript. DMH and LY designed the statistical plan. $L Z, Y G$, LY, YJH, LX, and HWZ participated in data acquisition and provided administrative, technical, or material support. All authors have read and approved the final manuscript and will take public responsibility for appropriate portions of the content.

\section{Funding}

This study was supported by a grant from the National Natural Science Foundation of China (81573787). The funder has no role in the design of the study and collection, analysis, and interpretation of data and in writing the manuscript.

\section{Declarations}

\section{Dissemination policy}

Findings will be published in peer-reviewed journals and presented at local, national, and international meetings and conferences to promote and explain the research to clinicians, patients, commissioners, and general medical community. We use the Clinical Trial Management Public Platform to manage and share our data. The data access is only for research, from January 2026 for 1 year.

\section{Ethics approval and consent to participate}

Central ethical approval has been confirmed from the Medical Ethics Committee of Tongji hospital affiliated to Tongji Medical College of Huazhong University of Science and Technology (TJ-IRB20190620). Written informed consent will be obtained from patients prior to enrolment.

\section{Consent for publication}

Not applicable.

\section{Competing interests}

The authors declare that they have no competing interests.

\section{Author details}

${ }^{1}$ Tongji Hospital, Tongji Medical College, Huazhong University of Science and Technology, 1095 Jiefang Avenue, Wuhan 430030, Hubei, China. ${ }^{2}$ First Hospital of Wuhan, 215 Zhongshan Avenue, Wuhan, Hubei, China. ${ }^{3}$ Union Hospital, 1277 Jiefang Avenue, Wuhan, Hubei, China. ${ }^{4}$ Reproductive Hospital, Jiangxi University of Traditional Chinese Medicine, 597 Shuangmashi Road, Nanchang, Jiangxi, China.

Received: 24 December 2020 Accepted: 30 November 2021 Published online: 14 December 2021

\section{References}

1. Chang G, Guiling F, Jian G, Yixue L, Yu L. Clinical analysis of patients with polycystic cystic ovary syndrome and ovarian reserve dysfunction when doing IVF-ET. Hebei Medl J. 2013;35(09):1333-4.

2. Coulson C, Jenkins J. Complementary and alternative medicine utilisation in NHS and private clinic settings: a United Kingdom survey of 400 infertility patients. J Exp Clin Assist Reprod. 2005;2(1):5. https://doi.org/10.1186/1743-1 050-2-5.

3. Smith JF, Eisenberg ML, Millstein SG, Nachtigall RD, Shindel AW, Wing H, et al. The use of complementary and alternative fertility treatment in couples seeking fertility care: data from a prospective cohort in the United States. Fertil Steril. 2010;93(7):2169-74. https://doi.org/10.1016/j.fertnstert.201 0.02 .054

4. Stankiewicz M, Smith C, Alvino H, Norman R. The use of complementary medicine and therapies by patients attending a reproductive medicine unit in South Australia: a prospective survey. Aust N Z J Obstet Gynaecol. 2007; 47(2):145-9. https://doi.org/10.1111/j.1479-828X.2007.00702.x.

5. Zhenhong S, Fang L. Advances in the application of acupuncture in assisted reproduction and its mechanism of action. J Tradit Chin Med. 2013;54(24): 2149-51.

6. Xi L, Qian L, Jie C, Qixin H, Zhengyun X, Pengyan Y, et al. A systematic review and meta-analysis of the effectiveness of acupuncture in treating premature ovarian failure. J Tradit Chin Med. 2016;57(12):1027-32.
7. Yong $\mathrm{P}$, Yanyan $\mathrm{H}$. Meta-analysis of the effect of acupuncture on premature ovarian failure. Clin J Trad Chin Med. 2016;28(1):108-12.

8. Hongfeng W, Xiaohong W. Zuoguiyin and erzhiwan plus or minus combined with acupuncture treating 60 cases of DOR patients. Shandong J Trad Chin Med. 2014;23(11):894-6.

9. Zhenru X. Effect of electro-acupuncture on ovarian reserve function improvement. Beijing: Beijing University of Chinese Medicine; 2015.

10. MacPherson H, Altman DG, Hammerschlag R, Youping L, Taixiang W, White A, et al. Revised STandards for Reporting Interventions in Clinical Trials of Acupuncture (STRICTA): extending the CONSORT statement. PLOS Med. 2010;7(6):e1000261. https://doi.org/10.1371/journal.pmed.1000261.

11. Kong J, Gollub R, Huang T, Polich G, Napadow V, Hui K, et al. Acupuncture de qi, from qualitative history to quantitative measurement. J Altern Complement Med. 2007;13(10):1059-70. https://doi.org/10.1089/acm.2007. 0524.

12. Beck AT, Epstein N, Brown G, Steer RA. An inventory for measuring clinical anxiety: psychometric properties. J Consult Clin Psychol. 1988;56(6):893-7. https://doi.org/10.1037/0022-006X.56.6.893.

13. Beck AT, Steer RA, Ball R, Ranieri W. Comparison of Beck Depression Inventories -IA and -II in psychiatric outpatients. J Pers Assess. 1996;67(3): 588-97. https://doi.org/10.1207/s15327752jpa6703_13.

14. William WK. Zung. A rating instrument for anxiety disorders. Psychosomatics. 1971;12(6):371-9. https://doi.org/10.1016/S0033-3182(71 )71479-0.

15. Zung WW. A self-rating depression scale. Arch Gen Psychiatry. 1965;12(1): 63-70. https://doi.org/10.1001/archpsyc.1965.01720310065008.

16. Hong SB, Lee NR, Kim SK, Kim H, Jee BC, Suh CS, et al. In vitro fertilization outcomes in women with surgery induced diminished ovarian reserve after endometrioma operation: Comparison with diminished ovarian reserve without ovarian surgery. Obstet Gynecol Sci. 2017;60(1):63-8. https://doi. org/10.5468/ogs.2017.60.1.63.

17. Roustan A, Perrin J, Debals-Gonthier M, Paulmyer-Lacroix O, Agostini A, Courbiere B. Surgical diminished ovarian reserve after endometrioma cystectomy versus idiopathic DOR: comparison of in vitro fertilization outcome. Hum Reprod. 2015;30(4):840-7. https://doi.org/10.1093/humrep/ dev029.

18. Chow S, Shao J, Wang H. Sample Size Calculations in Clinical Research. 2nd Ed. Chapman \& Hall/CRC Biostatistics Series. 2008. P. 89.

19. Li X, Xu H, Fang Y, Shang J, Yang H, Zhou X, et al. Acupuncture with regulating menstruation to promote pregnancy for diminished ovarian reverse: a prospective case series study (article in Chinese). China J Tradit Chin Med Pharm. 2017;37(10):1061-5.

20. Zhou L, Xia Y, Ma X, Tang L, Lu J, Tang Q, et al. Effects of "menstrual cyclebased acupuncture therapy" on IVF-ET in patients with decline in ovarian reserve. Zhongguo Zhen Jiu. 2016;36(1):25-8.

21. Manheimer E, Zhang G, Udoff L, Haramati A, Langenberg P, Berman BM, et al. Effects of acupuncture on rates of pregnancy and live birth among women undergoing in vitro fertilisation: systematic review and metaanalysis. BMJ. 2008;336(7643):545-9. https://doi.org/10.1136/bmj.39471.4304 51.BE.

22. Xie ZY, Peng ZH, Yao B, Chen L, Mu YY, Cheng J, et al. The effects of acupuncture on pregnancy outcomes of in vitro fertilization: a systematic review and meta-analysis. BMC Complement Altern Med. 2019;19(1):131. https://doi.org/10.1186/s12906-019-2523-7.

23. Spaeth RB, Camhi S, Hashmi JA, Vangel M, Wasan AD, Edwards RR, et al. A longitudinal study of the reliability of acupuncture deqi sensations in knee osteoarthritis. Evid Based Complement Alternat Med. 2013;2013:1-12. https://doi.org/10.1155/2013/204259.

\section{Publisher's Note}

Springer Nature remains neutral with regard to jurisdictional claims in published maps and institutional affiliations. 